\title{
Vibrations in AO control: a short analysis of on-sky data around the world
}

Caroline Kulcsár ${ }^{1}$, Gaetano Sivo ${ }^{1-2}$, Henri-François Raynaud ${ }^{1}$, Benoît Neichel ${ }^{3}$, François

Rigaut $^{3}$, Julian Christou ${ }^{4}$, Andres Guesalaga ${ }^{5}$, Carlos Correia ${ }^{6}$, Jean-Pierre Véran ${ }^{6}$, Éric

Gendron$^{7}$, Fabrice Vidal ${ }^{7}$, Gérard Rousset 7 , Tim Morris ${ }^{8}$, Simone Esposito ${ }^{9}$, Fernando

Quirós-Pacheco ${ }^{9}$, Guido Agapito ${ }^{9}$, Enrico Fedrigo ${ }^{10}$, Lorenzo Pettazzi $^{10}$, Richard Clare ${ }^{10}$, Riccardo Muradore ${ }^{11}$, Olivier Guyon ${ }^{12}$, Frantz Martinache ${ }^{12}$, Serge Meimon ${ }^{2}$, Jean-Marc Conan ${ }^{2}$

${ }^{1}$ L2TI, Institut Galilée, Université Paris 13, Villetaneuse, France

2 ONERA, The French Aerospace Lab, DOTA, Châtillon, France

${ }^{3}$ Gemini Observatory, La Serena, Chile

${ }^{4}$ Gemini Observatory, Hilo, Hawaii, USA

${ }_{5}^{5}$ Universidad Catolica de Chile, Center for Astro-Engineering, Santiago, Chile

${ }^{6}$ National Research Council, Herzberg Institute of Astrophysics, Victoria, Canada

7 LESIA, Observatoire de Paris, CNRS, Université Paris Diderot, UPMC, Meudon, France

${ }^{8}$ Durham University, Dep ${ }^{\mathrm{t}}$ of Physics, Durham, UK

${ }^{9}$ INAF, Osservatorio di Astrofisico di Arcetri, Firenze, Italy

${ }^{10}$ ESO, Garching, Germany

${ }^{11}$ University of Verona, Dep ${ }^{t}$ of Computer Science, Verona, Italy

${ }_{12}$ Subaru Telescope, National Observatory of Japan, Hilo, Hawaii, USA

\begin{abstract}
We present in this paper an analysis of several tip-tilt on-sky data registered on adaptive optics systems installed on different telescopes (Gemini South, William Herschel Telescope, Large Binocular Telescope, Very Large Telescope, Subaru). Vibration peaks can be detected, and it is shown that their presence and location may vary, and that their origin is not always easy to determine. Mechanical solution that have been realized to mitigate vibrations are presented. Nevertheless, residual vibrations may still affect the instruments' performance, ranging from narrow high frequency vibration peaks to wide low frequency windshake-type perturbations. Power Spectral Densities (PSDs) of on-sky data are presented to evidence these features. When possible, indications are given regarding the gain in performance that could be achieved with adequate controllers accounting for vibration mitigation. Two examples of controller identification and design illustrate their ability to compensate for various types of disturbances (turbulence, windshake, vibration peaks, ...), showing a significant gain in performance.
\end{abstract}

Keywords: Adaptive optics systems, vibration filtering, spectral analysis, tip/tilt control, atmospheric turbulence, disturbance identification, discrete-time LQG control, H2 control

\section{INTRODUCTION AND MOTIVATION}

Adaptive optics systems are known to compensate in real-time for optical aberrations caused by atmospheric turbulence, thanks to a deformable mirror (DM) that generates a correction wavefront computed from measurements obtained by a wavefront sensor (WFS). Yet other sources of perturbations may also degrade image quality; these include low temporal frequency signals induced by telescope tracking errors, or wind shaking of the telescope's structures that may also induce mechanical vibrations. When wind is present, the telescope's shutter and the vent gates, if any, can be closed in order to minimize the amount of wind inside the dome, but at the price of higher dome seeing (due to less ventilation). Other elements may cause vibratory effects, like fans,

Further author information: kulcsar@univ-paris13.fr

Adaptive Optics Systems III, edited by Brent L. Ellerbroek, Enrico Marchetti, Jean-Pierre Véran, Proc. of SPIE Vol. 8447, 84471C · (c) 2012 SPIE · CCC code: 0277-786/12/\$18 · doi: 10.1117/12.925984 

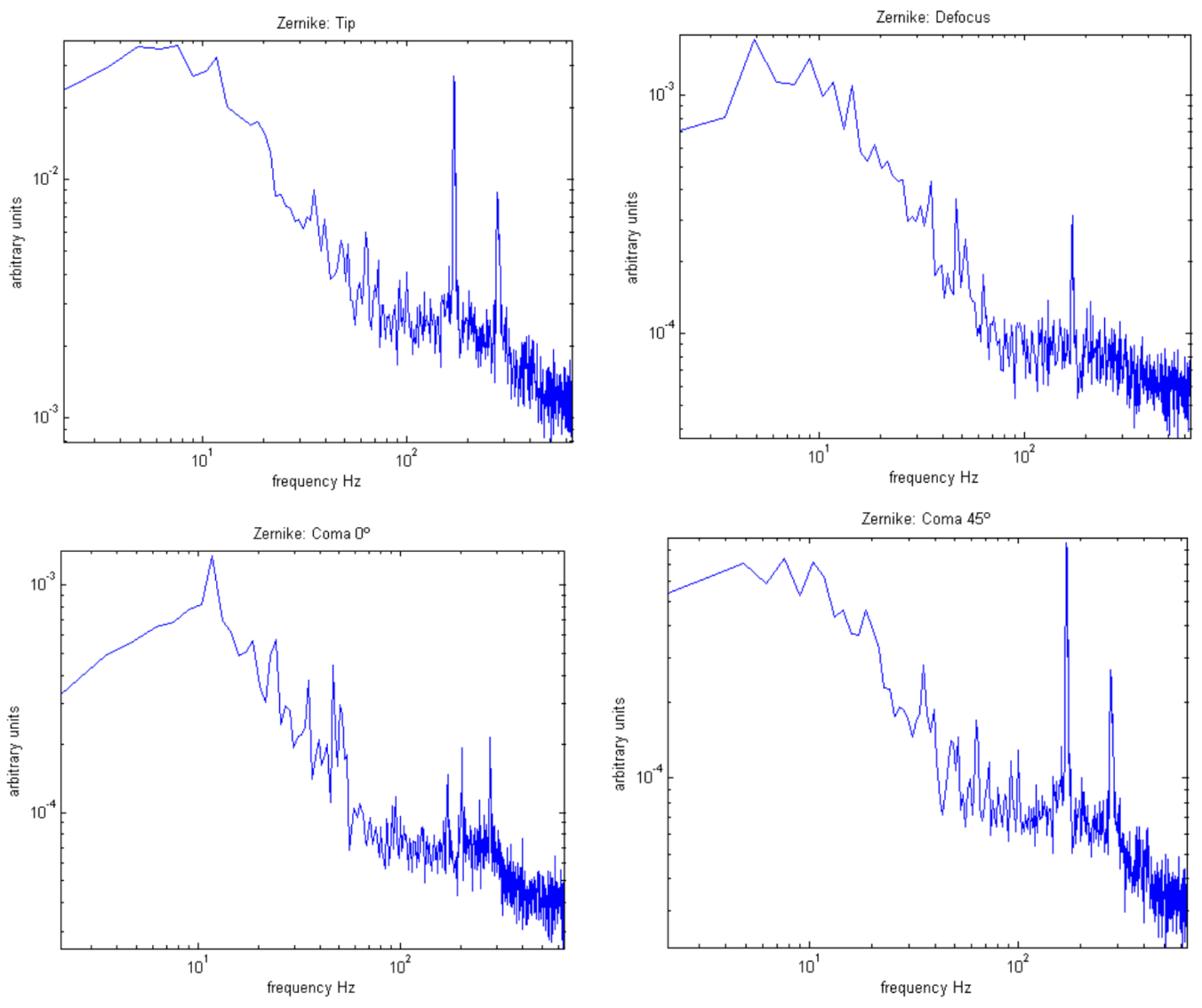

Figure 1. PSDs of tip, defocus and coma computed from NICI data. Frequency sampling is $1.3 \mathrm{kHz}$, and spectral resolution is $0.02 \mathrm{~Hz}$. Vibration peaks are here clearly visible on other modes than tip or tilt.

cryo-coolers and motors. Identifying the source of the vibration may be difficult, and usually requires extensive measurements and specific equipment such as accelerometers or dedicated WFSs.

Non-turbulent perturbations such as vibrating effects appearing in the optical path strongly affect the performance of the AO loop. These perturbations mainly affect tip and tilt components, but possibly higher modes, as first reported in NAOS at the VLT. ${ }^{1}$ Vibration peaks on higher modes are seen for instance in NICI (the Gemini Planet Imager's precursor at Gemini South), as illustrated by the power spectral densities (PSDs) in Figure 1. A strong peak around $170 \mathrm{~Hz}$ is detected on the tip, which is also seen on the defocus, coma and astigmatisms. The aim of this paper is to present some spectra of on-sky data for various sites, so as to illustrate the diversity of situations that can be encountered: Gemini South with GeMS data in Section 2, Gemini South with NICI and GPI in Section 3, the Canary MOAO demonstrator in Section 4, the Large Binocular Telescope in Section 5, the MACAO system at the VLT in Section 6, and the SCExAO system at Subaru Telescope in Section 7.

It is obvious that vibrations should be, when feasible, mitigated thanks to mechanical actions, see in particular Section 7. Nevertheless, remaining vibrations and/or windshake effects may still affect performance, and these can be attenuated thanks to adequate controllers, as proposed in, e.g., Refs. 2-11. Two examples of such controllers are presented in Section 8 corresponding to Refs. 4 and 10.

\section{THE MCAO SYSTEM GEMS AT GEMINI SOUTH}

GeMS is the Gemini's multi-conjugate AO (MCAO) system. It uses 5 artificial Laser Guide Stars (LGSs) with their associated LGS WFSs and 3 DMs to compensate the turbulence over a field of view of 2 arcmin. Besides 
this, 3 Natural Guide Stars (NGSs) are required for the control of the tip-tilt and plate scale modes. GeMS started on-sky commissioning in January 2011. Since then on-sky data from NGS WFSs and LGS WFSs (in open and closed-loop) were gathered to characterize the vibration environment seen by GeMS.

The AO bench is mounted at the Cassegrain focus of the telescope. In an effort to reduce the potential vibration sources, the electronic cabinets are mechanically decoupled from the AO bench itself. However, as all the telescope instruments are mounted on the same mechanical structure (called Instrument Support Structure: ISS) vibrations can be transmitted from one instrument to another. In particular, one of the main sources of vibrations that has been identified for GeMS founds its origin in the cryo-coolers of the Near-Infrared imager (called GSAOI). These cryo-coolers induce a peak around $55 \mathrm{~Hz}$, which is particularly affecting the Y-axis (Tilt mode) of GeMS WFSs. We measure the amplitude of this vibration to be of the order of 5 to 10 mas rms, which would reduce the Strehl by approximately $10 \%$ in H-band. At lower frequency, we do also see a broad peak around $14 \mathrm{~Hz}$, which is believed to be induced by the top-end of the telescope, and the secondary mirror structure. This peak is not always present and may be excited by wind shake.

The data presented in Figure 2 have been acquired in open-loop with the LGS WFS and the NGS WFS. The tip-tilt signal coming from the LGS WFS cannot be used directly, as the up-link tip-tilt of the laser is compensated by fast tip-tilt platforms in the laser projection system. Hence we used LGS WFS data when the up-link and the NGS WFS loop were open. This may affect the amplitude of the vibration, as the linearity range of the LGS WFSs is only of the order of few arcsecs, but this should not affect the frequencies at which vibrations are detected. The LGS data have been acquired at $450 \mathrm{~Hz}$. For the NGS WFSs, data were acquired while the LGS loop was closed in order to get smaller spots. The NGS data has been acquired at $600 \mathrm{~Hz}$. We notice the presence of a peak at $55 \mathrm{~Hz}$, but its energy is lower due to the mode of acquisition (up-link and NGS WFS loops open). Advances controllers as the one presented in Section 8.2 are envisioned to compensate for these perturbations on tip and tilt modes.

\section{NICI AND GPI AT GEMINI SOUTH}

In the context of designing the Gemini Planet Imager (GPI), the GPI team tried to understand the vibration environment at Gemini South by processing telemetry data acquired with GPI's precursor NICI. These are closed loop $1.3 \mathrm{kHz}$ telemetry data of measured tilt obtained in 2008, from which open loop telemetry data are reconstructed. Our method and findings are reported in Ref. 12. We found that vibration content at low temporal frequencies $(<60 \mathrm{~Hz})$ could not be evaluated, due to spatial aliasing effects on NICI's curvature WFS. However, we found significant vibration peaks at high frequencies as shown in Figure 3. Such high frequency vibrations most likely originate from NICI itself, rather than from the Gemini telescope. However, if occurring in GPI as well, they would be beyond the correction capabilities of GPI, even at a $2 \mathrm{kHz}$ AO frame-rate, with a simple integrator with adjustable gain, which was the baseline controller. Such vibrations would significantly compromise the performance of the GPI instrument.

In a subsequent study, we have assessed how such high frequency vibrations could be mitigated with a more advanced LQG-based minimum variance (MV) controller. We only used the three main vibration peaks $(81 \mathrm{~Hz}$, $170 \mathrm{~Hz}$ and $279 \mathrm{~Hz}$ ) from Figure 3, and assumed that the one centred at $170 \mathrm{~Hz}$ was in the non-common path (NCP) (i.e. it only affected the WFS path, not the science path). We built a hybrid continuous-discrete time model to assess and compare the performance of two different controllers: a classical integrator controller with adjustable gain ("Type-I") and an MV controller with vibration suppression embedded. ${ }^{9}$ The MV controller uses auto-regressive (AR) models of different orders, up to order 3.

Using discrete-time transfer-functions, we computed theoretical residuals, and compared them with the empirical residuals obtained from the output of our hybrid model (E2E simulation), which was fed with random time series of tilt errors, corresponding to the expected median observing conditions for GPI, ${ }^{5}$ superimposed with the high frequency vibrations lines.

Figure 4 shows that the MV controller provides a dramatic performance improvement, with little advantage of using AR model of order 3 or more. As regards NCP aberrations, sign ' + ' is used when the controller attempts to reject the NCP vibration and '-' when it does not. In the latter case, the $170 \mathrm{~Hz}$ vibration is simply not simulated. It is very interesting to see that the MV controller is able to reject very efficiently the NCP vibration when present, regardless of the order of the AR model. 


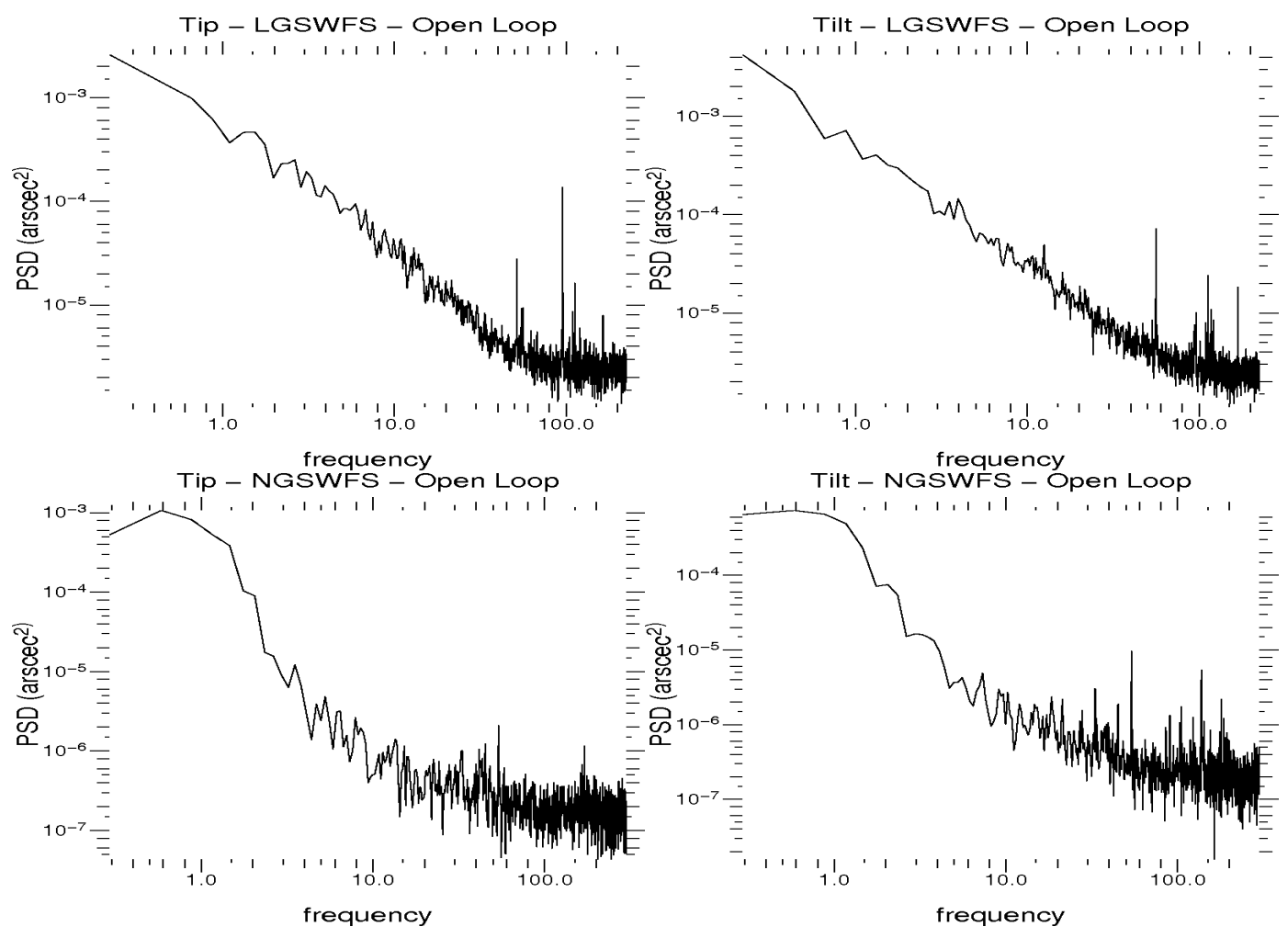

Figure 2. DSPs of LGS WFS (top) and NGS WFS (bottom) data in open-loop. The peak at 55 Hz does not impact the data in a similar way. Sampling frequency and spectral resolution are $450 \mathrm{~Hz}$ and $0.02 \mathrm{~Hz}$ for LGS WFS, and $600 \mathrm{~Hz}$ and $0.08 \mathrm{~Hz}$ for NGS WFS.
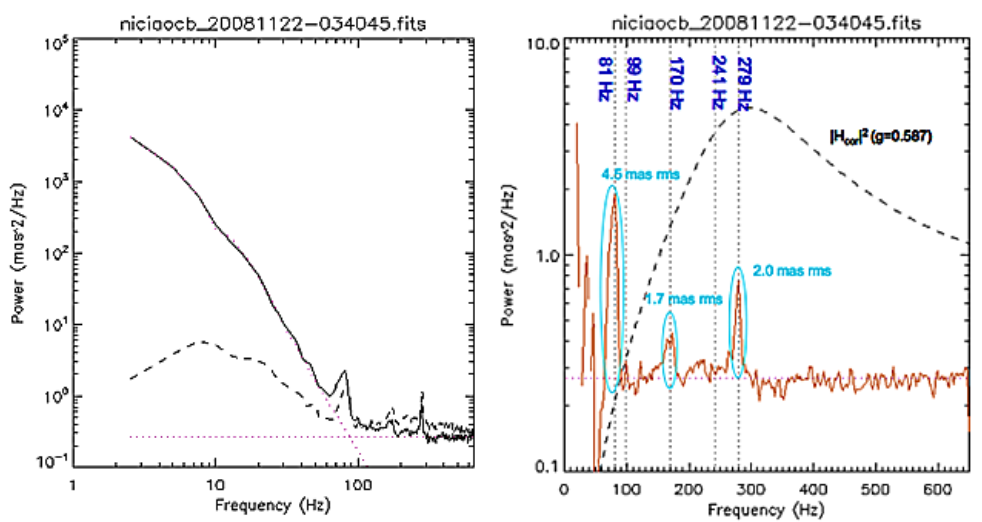

Figure 3. Left: reconstructed incoming T/T PSD (solid line) from the NICI residual PSD (dashed line) given by the telemetry data. The dotted green lines represent the estimated low frequency component and the estimated noise level. Right: high frequency component (blue line) super-imposed with the GPI rejection transfer function at a frame rate of $2 \mathrm{kHz}$ (dashed line). Figure reproduced from Véran et al, Ref. 12 


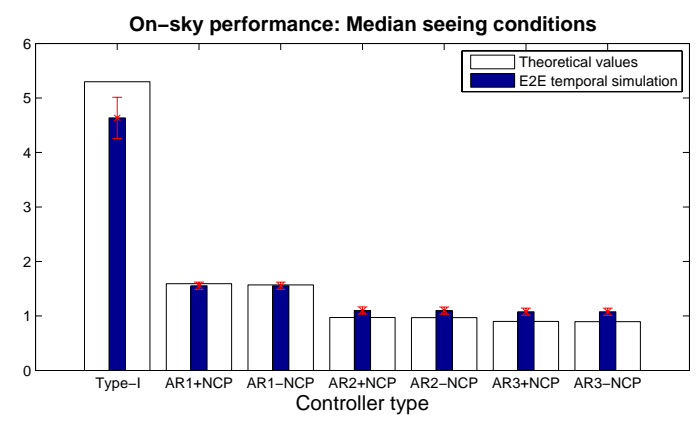

Figure 4. Simulated on-sky performance obtained for the median-seeing case. Note the good agreement of theoretical and sample residuals for all the LQG controllers. (Figure reproduced from Correia et al, Ref. 13)

\section{THE CANARY MOAO DEMONSTRATOR AT THE WILLIAM HERSCHEL TELESCOPE}

Canary is a demonstrator instrument of multi-object adaptive optics (MOAO) technique. It is installed at the Nasmyth focus of the William Herschel Telescope, in the Canary islands. It is equipped with 3 NGS WFSs looking at 3 stars taken in a field of view of 2.5 arcmin diameter. First MOAO-compensated images have been obtained end of 2010. ${ }^{14}$ A wealth of wave-front data have been acquired with Canary, and allowed us to study vibration effects. The power spectral densities shown here are all obtained using Welsh method, cutting the data

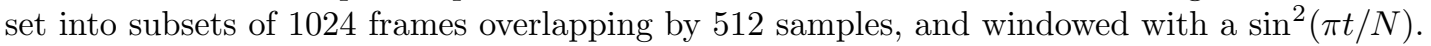

Figure 5 shows the periodogram where vibrations are particularly visible. Data have been taken in the night of Sept. 20th, 2010. Two synchronized wave-front sensors looking in different directions of the field (33 arcsec apart) were simultaneously acquiring the turbulence data, so that we can also plot the power spectral density of the difference, i.e. of fluctuations that were not common to both WFSs. The gap between the two curves helps to reveal the vibration peaks. A vibration along $y$ axis ("vertical" axis, with respect to the optical table) at $47 \mathrm{~Hz}$
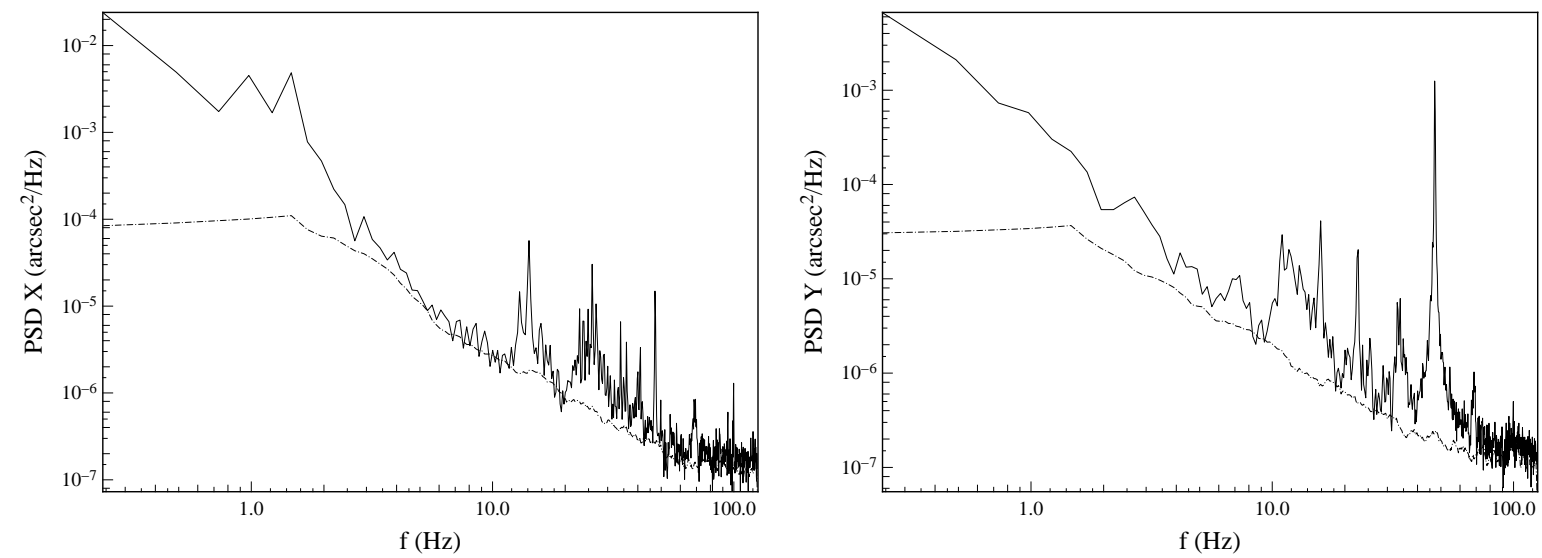

Figure 5. Solid lines: Power spectral density (periodogram) of tilt along $x$ (left graph, $x$ is horizontal with respect to the Nasmyth optical table) and $y$ (right, vertical) axis obtained with Canary. Sampling frequency was $250 \mathrm{~Hz}$. Dashed line: fluctuations not common to both wave-front sensors data. Spectral resolution is $0.24 \mathrm{~Hz}$.

was present during all the night. Three different sampling frequencies of 100,175 and $250 \mathrm{~Hz}$ were used during the night. Of course the vibration is smoothed (convolved) by the integration time, and appears with a smaller amplitude when recorded with low sampling frequencies. The amplitude is 0.074 " rms (integrated between 46 and $48 \mathrm{~Hz}$ ) which means a peak-to-peak amplitude of $0.20 "$. 
Others broad features are present on the spectrum, centered around $10 \mathrm{~Hz}$, and around $25 \mathrm{~Hz}$, with a total width of about $10 \mathrm{~Hz}$. Although clearly visible on the PSDs, they only account for 0.014 arcsec rms each (0.02" peak-to-peak). Figure 6 shows series of results obtained in the night of Sept. 27th, 2010. All of them have been
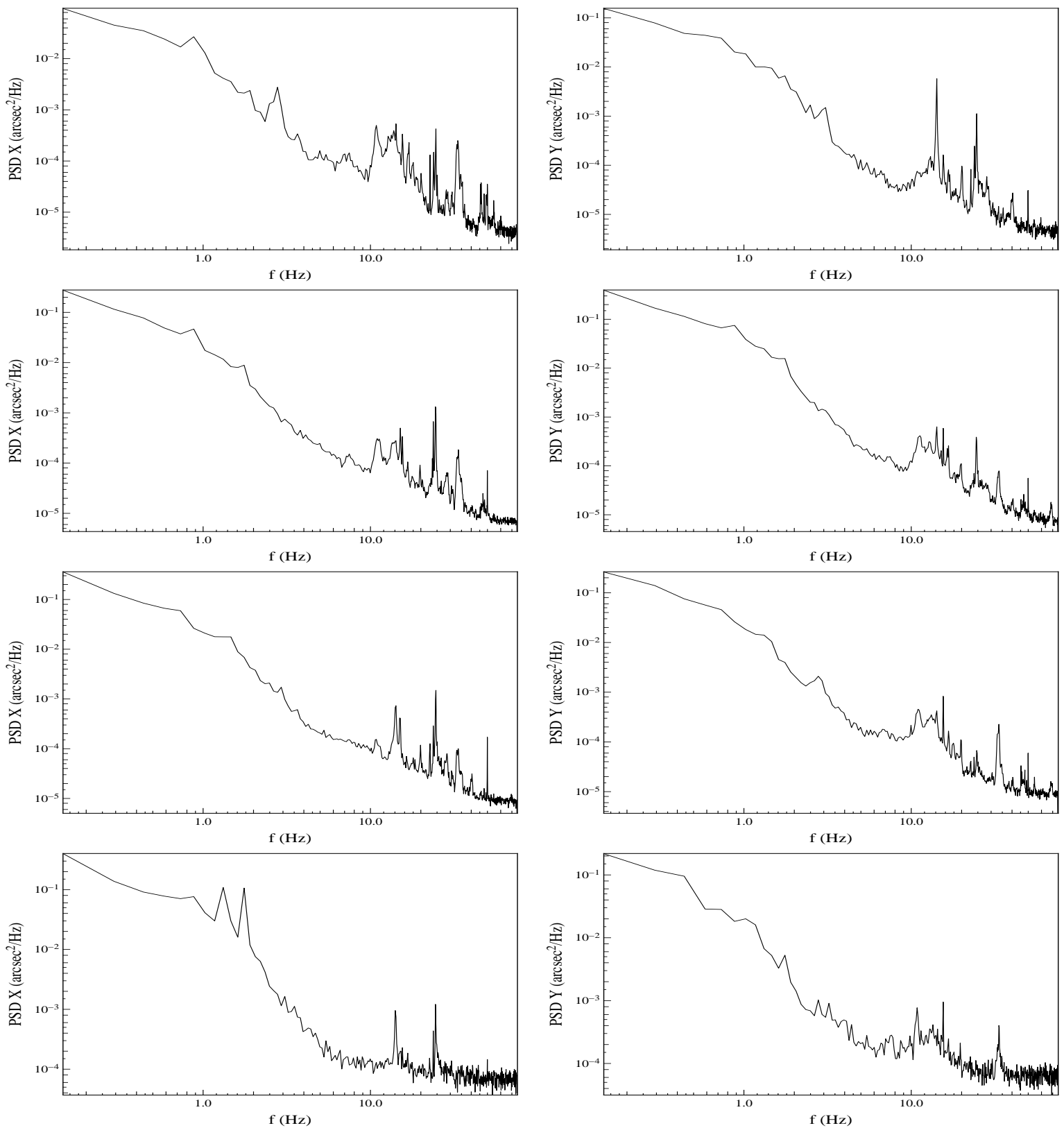

Figure 6. Power spectral density of tilt along $x$ (left graph, $x$ is horizontal with respect to the Nasmyth optical table) and $y$ (right, vertical) axis obtained with Canary on Sept. 27th, 2010. Sampling frequency is 150 Hz. From top to bottom, data sets acquired at 23h59, 1h34, 2h33 and 6h58. Spectral resolution is $0.15 \mathrm{~Hz}$.

acquired with a sampling frequency of $150 \mathrm{~Hz}$. They correspond to data sets acquired (from top to bottom) at 23h59, $1 \mathrm{~h} 34,2 \mathrm{~h} 33$ and $6 \mathrm{~h} 58$. The line at $47 \mathrm{~Hz}$ has disappeared. Series of vibration peaks are visible, ranging from 10 to $60 \mathrm{~Hz}$. On both axes, features at 14 and $25 \mathrm{~Hz}$ remain present during the whole night. A $11 \mathrm{~Hz}$ vibration peak is also present, but with variable energy, and with some transfer between $x$ and $y$ axes. Small 
low frequency features appear here and there: at $0.9 \mathrm{~Hz}$, most of the time on the $x$ axis, except at $2 \mathrm{~h} 33$ where it seems to be transferred to the $y$ axis, and at $\sim 1.9 \mathrm{~Hz}$ with the same characteristics. The total of the energy contained in these peaks is equivalent to a vibration with an amplitude of 0.03 to 0.07 arcsec rms (0.1 to 0.2 arcsec peak-to-peak) on each axis, which remains hopefully pretty low.

Eventually, the Canary demonstrator will feature a full LQG controller including vibration filtering ${ }^{8}$ for the next on-sky runs in summer 2012, allowing to evaluate its performance in this context.

\section{FIRST LIGHT AO AT THE LARGE BINOCULAR TELESCOPE}

In the case of the Large Binocular Telescope ${ }^{15}$ (LBT), the swing arm supporting the Deformable Secondary Mirror (DSM) has a resonance frequency at $\sim 13 \mathrm{~Hz}$. Wind shaking and telescope tracking can excite this resonance frequency producing an overall open-loop image jitter of $\sim 26$ mas rms (Figure 7). The temporal controller of the First Light AO (FLAO) system is based on a modal integrator in which Tip/Tilt (TT) gains can be optimized with an automatic procedure that sweeps a range of gain values and selects the value minimizing the residual TT variance. ${ }^{16}$ Under high-flux conditions, when the FLAO system is operated at fast frame rates (fs $>800 \mathrm{~Hz}$ ), the $13 \mathrm{~Hz}$ vibration lies within the $\mathrm{AO}$ correction bandwidth and so it can be partially attenuated. We have characterized the loss in performance due to residual TT jitter caused by this vibration. For instance, a maximum SR in $\mathrm{H}$ band of $80 \%$ can be dropped by a factor 2 in the presence of a residual jitter of $\sim 10$ mas rms. ${ }^{17}$ Observed-based control algorithms better suited to deal with telescope vibrations have been also investigated but not implemented on-sky as yet. ${ }^{7}$
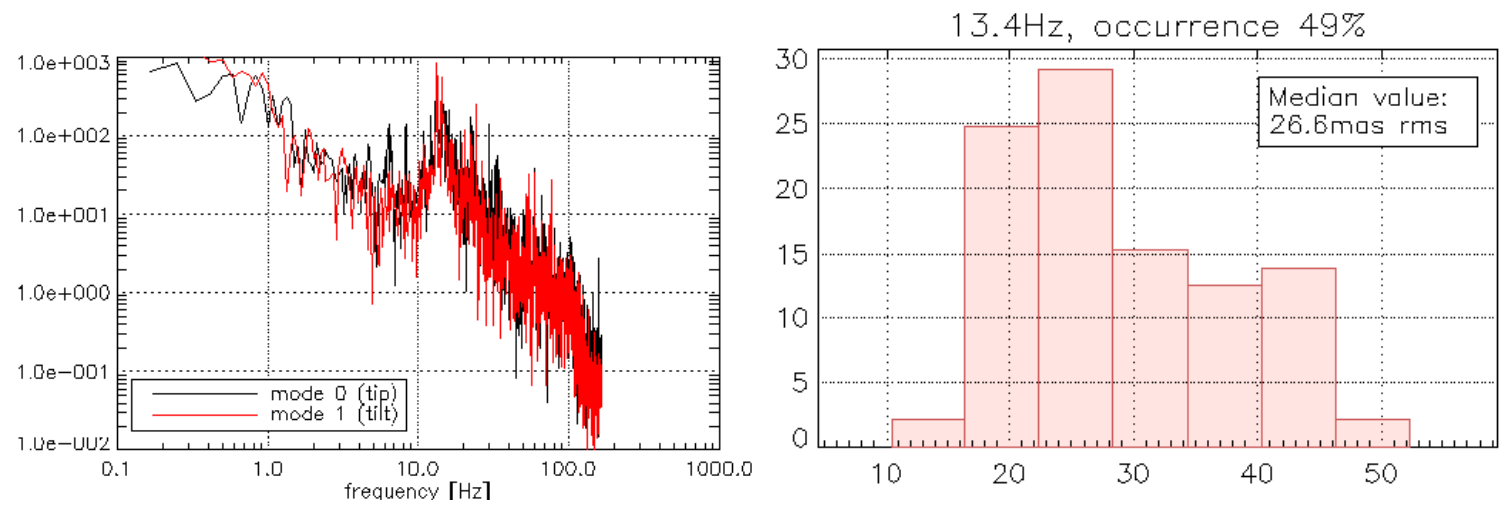

Figure 7. Left: example of open-loop TT spectra estimated from AO-telemetry data. The strong telescope vibration centered at $\sim 13 \mathrm{~Hz}$ is clearly seen. Right: histogram (mas rms) of the $13.4 \pm 0.1 \mathrm{~Hz}$ vibration present on TT modes estimated from the reconstructed open-loop data. The percentage of occurrences is computed with respect to the total of 282 analyzed data sets acquired during the commissioning of the FLAO 1 system.

\section{THE MACAO SYSTEM AT THE VLT}

MACAO (Multiple Application Curvature Adaptive Optics system) is a 60 element AO system operating at the Coudé focus of the VLT's unit telescopes to serve the VLT Interferometer as well as the AO module used in SINFONI and CRIRES. The MACAO-VLTI installation and on-sky verification took place in 2003-2004 followed by the SINFONI and CRIRES modules. Since then the MACAO-VLTI system is operating fulfilling its objective of delivering a corrected wavefront to the VLTI delay lines.

Nevertheless during the first years of operation a number of problems that had a deleterious impact on the performance of the interferometer served by the MACAO system could be identified, amongst which were vibrations. In the effort of optimizing the quality of the wavefront delivered to the delay lines, a series of interventions have been implemented that culminated in the removal of the cabinets from the Coudé focus. As a result of these interventions the performance of the system could be substantially improved resulting in a 
beneficial impact on the VLTI fringe tracking (see Ref. 18 for a complete analysis of all the problems identified and a detailed discussion of these results).

However the system is not yet free from the detrimental effect of vibrations. Figure 8-left shows the PSD of tip/tilt residuals of the MACAO system sampled at $420 \mathrm{~Hz}$. The PSD is computed with the Welch method over a time window of $1 \mathrm{~min}$. The plots clearly show narrow-band peaks appearing especially in the frequency region around $50 \mathrm{~Hz}$. Considerable work is currently being undertaken in order to precisely identify the sources of these vibrations.

The AO controller of MACAO could be upgraded with vibration rejection algorithms in order to further optimize the closed loop performance. An optimistic estimate of the gain in performance that can be achieved by mitigating narrow-band perturbations can be obtained by processing the closed loop tip/tilt residuals with sharp band-stop filters ${ }^{11}$ (for example having $1 \mathrm{~Hz}$ bandwidth and $20 \mathrm{~dB}$ peak reduction). The results of this analysis are shown in Figure 8-right where the PSD of the tip residuals after processing with band-stop filters at $45 \mathrm{~Hz}$ and $48 \mathrm{~Hz}$ are displayed. The suppression of the vibrations at $45 \mathrm{~Hz}$ and $48 \mathrm{~Hz}$ yields an improvement of performance in the order of $8 \%$ in terms of tip residual rms.
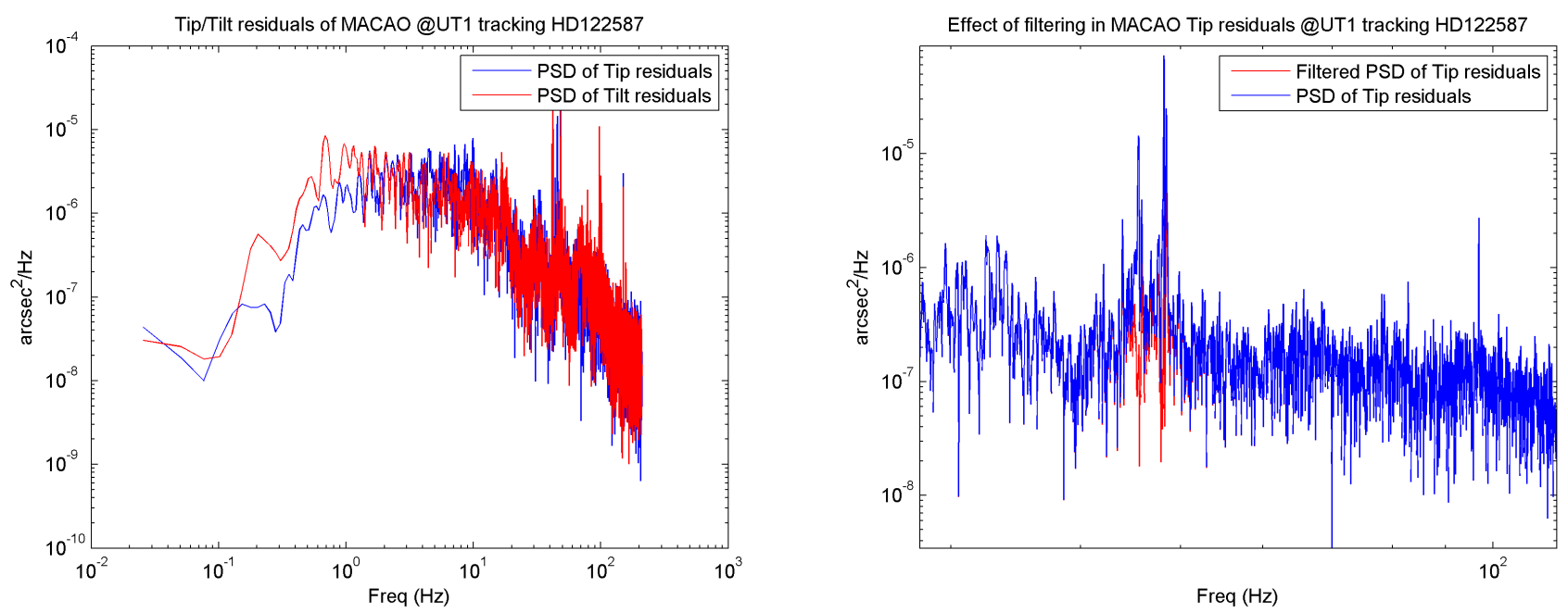

Figure 8. Left: PSD of closed loop MACAO tip/tilt residuals. Right: Filtered vs. non-filtered PSD of closed loop MACAO tip residuals. Spectral resolution is $0.02 \mathrm{~Hz}$.

\section{THE INSTRUMENT SCEXAO AT SUBARU TELESCOPE}

The Subaru Coronagraphic Extreme Adaptive Optics (SCExAO) is a high contrast imaging instrument currently under development on the Subaru $8.2 \mathrm{~m}$ Telescope. SCExAO is installed downstream of the 188-actuator facility adaptive optics system, and applies further wavefront control using techniques that are optimized for high contrast imaging, such as focal plane speckle sensing in the near-IR and non-linear curvature wavefront sensing in visible light. The uniqueness of SCExAO is its ability to image companions very close to the optical axis (down to $\sim 1 \lambda / D$ ), made possible by the use of a PIAA-type coronagraph. A review of the SCExAO system can be found in a separate paper in this conference. ${ }^{19}$

SCExAO is unusually sensitive to vibrations, due to the small inner working angle of its coronagraph, which is very unforgiving for tip-tilt aberrations. Tip-tilt in SCExAO needs to be calibrated to milliarcsecond level, and should be actively controlled to be within a few milliarcsecond rms. Early on-sky tests revealed that tip-tilt in SCExAO was initially around 10 mas rms, about one order of magnitude higher than required. Multiple frequency peaks were observed, see Figure 9, using fast frame imaging with SCExAO's cameras. A complete understanding of the sources of vibrations proved to be very challenging, partially because the tip-tilt measured by the visible ( $1 \mathrm{kHz}$ frame rate) and IR ( $70 \mathrm{~Hz}$ frame rate) cameras were not completely consistent, showing that 
vibrations were exciting elements in the non-common path between the two arms of the instrument. Attempts to link vibrations to accelerometer measurements also proved to be very challenging. Multiple frequencies above $100 \mathrm{~Hz}$ were observed, and their active compensation would be challenging with the SCExAO hardware.

Our approach to mitigate vibrations and their effects were to:

1. Mechanically strengthen optical mounts that were deemed to be the weakest. Identification of weak points was done in the lab by applying forces to mounts and monitoring image motion. This reduced SCExAO's sensitivity to vibrations.

2. Insert a layer of elastomer within SCExAO's 3 mounting points. This proved to be very successful, see Figure 9, and was easily done since SCExAO is in a fixed gravity environment. Given this success, we also applied the same passive vibration isolation on the HiCIAO near-IR camera located downstream of SCExAO, as HiCIAO's cooler was a major source of vibrations for our instrument.

3. Use a high sensitivity coronagraphic low-order wavefront sensor to measure in real-time pointing of the coronagraph to milliarcsecond accuracy. This sensor, the coronagraphic low-order wavefront sensor (CLOWFS), is described in a previous publication. ${ }^{20}$ It uses light reflected by an annulus imprinted on the coronagraph focal plane mask, and has been demonstrated to measure tip-tilt to $10^{-3} \lambda / D$ in the lab (equivalent to 0.05 mas for SCExAO). Further tests at NASA JPL have demonstrated $3.10^{-4} \lambda / D$ closed loop tip-tilt control in visible light with this sensor. We have operated a near-IR version of this sensor on-sky with the SCExAO instrument to measure and correct for tip-tilt. With $70 \mathrm{~Hz}$ sampling, the sensor is a very effective complement to approach (2), which tends to suppress high frequency vibrations but leaves low frequency (up to $\sim 10 \mathrm{~Hz}$ ) vibrations.

4. Use the CLOWFS described above to calibrate the impact of vibrations on the coronagraphic science image. The CLOWFS logs tip-tilt errors, that are then used to reconstruct a simulated focal plane image containing coronagraphic leaks due to vibrations. The approach is described in Ref. 21, and was demonstrated to offer one to two orders of magnitude in contrast improvement when the contrast limit is due to vibrations.

Together, these four techniques have reduced the effect of vibrations to a level such that they no longer limit the instrument's performance. We are currently implementing a red/visible version of the CLOWFS in SCExAO, able to measure tip-tilt more accurately and also more rapidly ( $1 \mathrm{kHz}$ sampling). An LQG-type controller is also being implemented to better control vibrations, using both the visible and near-IR CLOWFS signals.
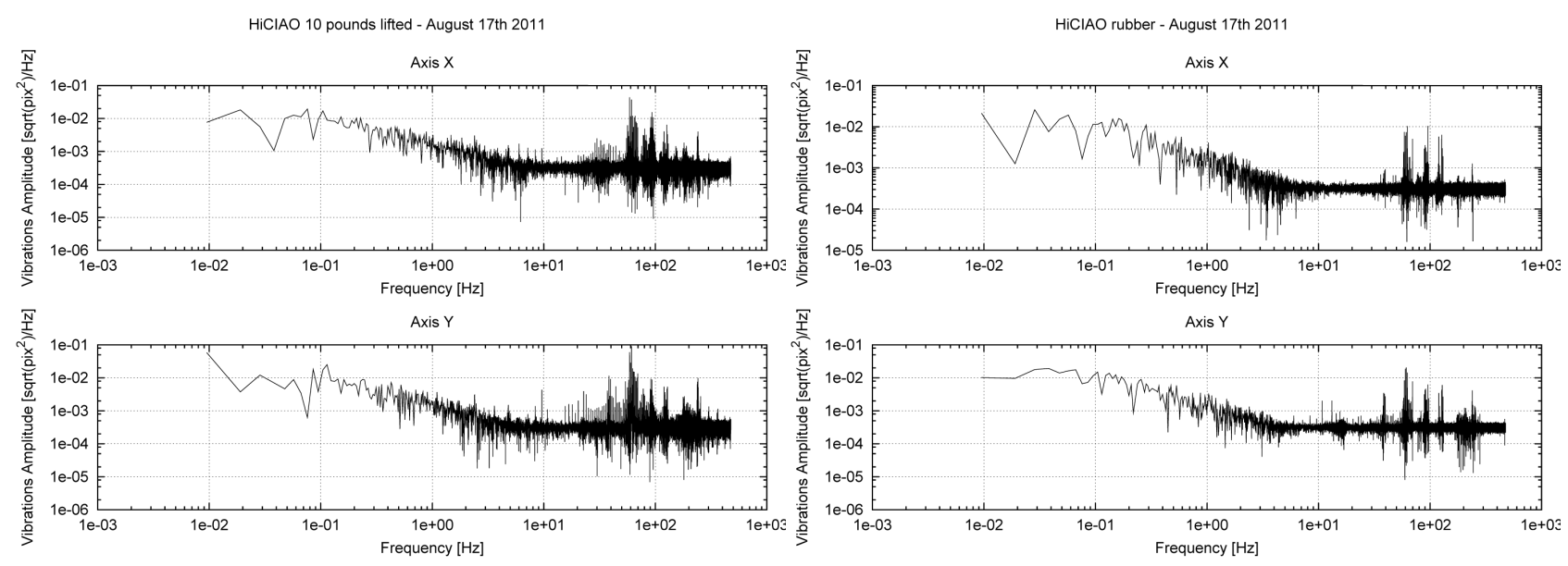

Figure 9. PSDs before (left) and after (right) the rubber step 2. Frequency sampling is $1 \mathrm{kHz}$. 


\section{TWO EXAMPLES OF CONTROLLER SYNTHESIS}

Substantial gains in performance can be achieved with advanced controllers when they are properly designed to reduce the effect of external disturbances such as vibrations. ${ }^{2,5,9}$ However, large errors in the subsequent modelization may severly reduce the gains in performance provided by these sophisticated controllers. In some cases, they could even show no clear difference to the classical integrator. This emphasizes the need for on-line identification or tuning procedures that would ensure good performance. ${ }^{3,4,7,10-12,22}$ Two example of modelbased controllers are proposed: an LQG design based on a perturbation model identified from open-loop data spectrum as proposed in Ref. 4, and an $\mathrm{H}_{2}$ design with parameters identified using a criterion on the closed-loop spectrum as proposed in Ref. 10.

\subsection{Controller design by identification of the perturbation's PSD}

The idea underlying the method in Ref. 4 is to use a disturbance model as close as possible to the actual physical process. Indeed, the physical description of a phenomenon involves parameters chosen because they are easy to interprete, as they avoid some flaws affecting more formal descriptions: a slight variation of the parameter does not induce a huge change in the phenomenon amplitude; the dimension and physical meaning of each parameter are clear; independent processes are described with independent parameters. These properties tend naturally to ease the optimization process underlying the identification of a model based on such physically meaningful parameters.

Therefore, each contributor of the disturbance signal (turbulent component, each vibration peak) is described as an independent mechanical oscillatory system of the second order. This leads to a state-space description with independent auto-regressive processes of order 2 (AR2) in parallel. Each AR2 process $\{x\}$ can be described under the form

$$
x_{k+1}=a_{1} x_{k}+a_{2} x_{k-1}+\sigma \epsilon_{k}
$$

where $\{\epsilon\}$ is a normalized zero-mean Gaussian white noise. To keep close to the physical description, the parameters identified are not the $\left(a_{1}, a_{2}\right)$ of each component, but the central frequency and the damping coefficient of the corresponding mechanical oscillatory process. This on-line identification method makes use of a temporal sequence of WFS measurements (squared difference between data and model) to estimate an open-loop spectrum $\hat{S}$; the model parameters $\boldsymbol{p}$ are fitted by minimizing a maximum likelihood metric between this estimated openloop spectrum $\hat{S}$ and a synthetic spectrum $S(\boldsymbol{p})$ (computed from the parameters value):

$$
\hat{\boldsymbol{p}}=\operatorname{argmin}_{\boldsymbol{p}}\|\hat{S}-S(\boldsymbol{p})\|^{2} .
$$

It is run periodically as new batches of data are collected, in order to re-tune the filter parameters along observation as disturbance statistics evolve. Only two user defined parameters are required: the minimum and maximum frequency between which the vibration peaks are to be found (default settings are proposed). An originality of the method is to make use of the disturbance spectrum to identify sequentially the vibration peaks, in decreasing order of energy. The outputs of the method are (i) a model of the "smooth" component of the disturbance, e.g. wind shake and turbulence; (ii) a model of the "sharp" components, i.e. the vibration peaks, to be used in a state space framework with an LQG control scheme.

On the example given in Figure 10, the variance of the open-loop y-slopes measurements is $3193.810^{-6} \operatorname{mas}^{2}$. The variance of the residual y-slopes with the identified model used for LQG control is $260.510^{-6} \operatorname{mas}^{2}$. The improvement is due to vibration compensation, but also to a better fit of the other sources of disturbances (turbulence, windshake, ...).

\subsection{Controller design by minimization of closed-loop residuals}

In this second example, controllers are synthesized with $\mathrm{H}_{2}$ methods (see Ref. 23). These frequency techniques can readily take tip/tilt mirror loop dynamics and performance requirements into account within the design stages. Three controllers are tested for a later implementation in GeMS, at the Gemini Observatory: integrator with fixed gain, integrator with gain optimized to give the minimum residual variance, and $\mathrm{H}_{2}$ controllers that also minimize the residual phase variance, see details in. ${ }^{10}$ 

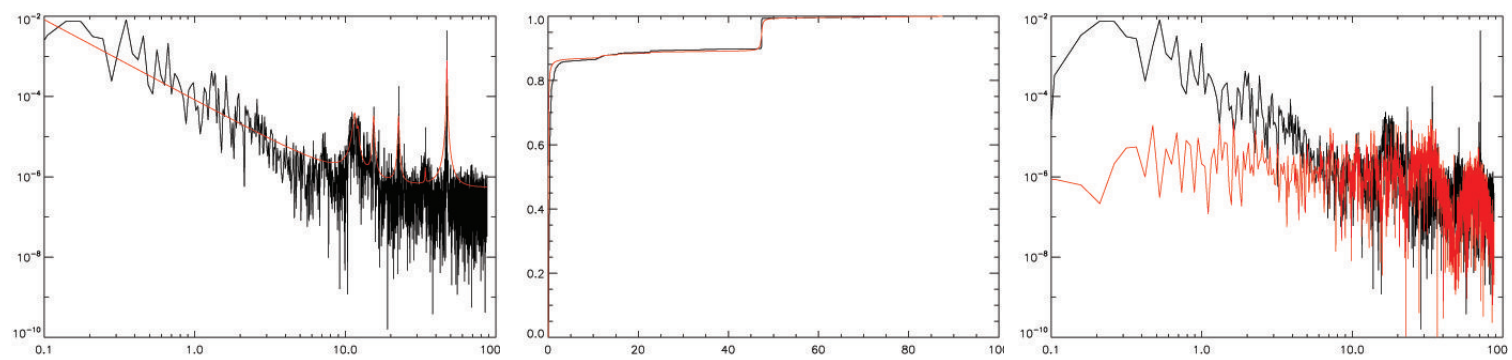

Figure 10. Example on Canary tilt data (20 sept. 2010). Left: perturbation's PSD (black curve) and total estimated PSD $\hat{S}$ (red) at the last step of the sequential identification process where peaks are estimated in decreasing order of energy. Middle: cumulated PSD of model (red) and perturbation (black), where the main peak is evidenced at $47 \mathrm{~Hz}$. Right: PSD of the residuals (red) compared to the PSD of the perturbation (black). Frequency sampling is $175 \mathrm{~Hz}$ and spectral resolution is $0.04 \mathrm{~Hz}$.

$H_{2}$ controllers are derived from the minimization of the $H_{2}$ norm of a performance output. ${ }^{23}$ The next objective here is to find the $\mathrm{H}_{2}$ controller $G(s)$ which also achieves

$$
\min _{G(s)} \sum_{k=0}^{N-1}\left|e_{k}\right|^{2}
$$

where $e_{k}$ is the residual phase. This departs from traditional approaches ${ }^{3,4,7}$ where model identification tools are used to represent the turbulence and vibrations, with a subsequent controller design based on this model. The identification step is here skipped and we look for a controller that starts from a basic control law, a "leaky" integrator:

$$
W_{e}(s)=\frac{C_{0}}{s+C_{1}} .
$$

The controller complexity is then increased (if needed) by adding filters with the pre-defined structure

$$
F(s)=\frac{s^{2}+2 \eta_{1} \omega_{o} s+\omega_{o}^{2}}{s^{2}+2 \eta_{2} \omega_{o} s+\omega_{o}^{2}} .
$$

At each step, the parameters of the newly added filter are optimized to give the minimum residual variance. This approach is not optimal but it sequentially constructs a controller with increasing complexity up to a point where no further improvement in the closed-loop performance is obtained. The method consists in scanning the spectrum within the range where vibrations are likely to exist, i.e. sweeping the frequency $\omega_{o}$ over the spectrum for several values of $\eta_{1}$ and $\eta_{2}$. This process is shown in Figure 11 (left), where function $F(s)$ (top) is swept from left to right, generating the corresponding variance plot (bottom). The data correspond to GeMS NGS WFS tilt measurements, and the open-loop variance drops from $1.2710^{-3} \operatorname{arcsec}^{2}$ to $0.40310^{-3} \operatorname{arcsec}^{2}$ with the $\mathrm{H}_{2}$ controller composed of 1 leaky integrator and 2 notches. From the implementation point of view the time required to execute a complete sequence is less than 3 minutes using a commercial PC. This processing time, although adequate for the dynamics of turbulence, would represent an excessive burden for the real time controller, so a stand-alone computer would be preferable in case of a practical implementation.

\section{CONCLUSIONS}

Vibrations are here to stay! They affect low order modes, most often tip and tilt but not only, as illustrated in the spectra of the introductory section. As seen in the various DSPs shown above, they might be present at different levels of energy, mixed with other kinds of perturbations, like windshake. As indicated in the introduction, a number of vibration mitigation methods have been proposed recently in $\mathrm{AO}$, showing a growing interest especially in the perspective of newly designed AO instruments. Two examples based on Refs. 4,10 have been proposed in Section 8 to illustrate the use of model-based controllers for tip/tilt mitigation, together with an identification 

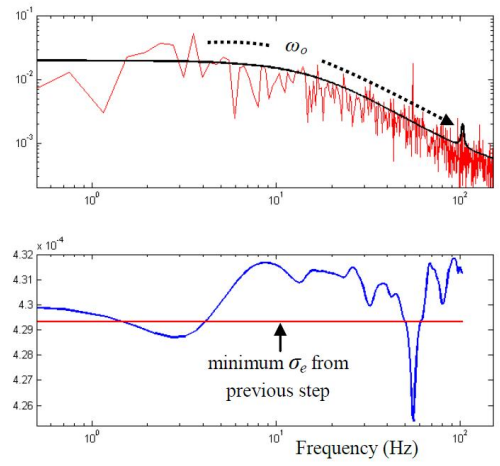
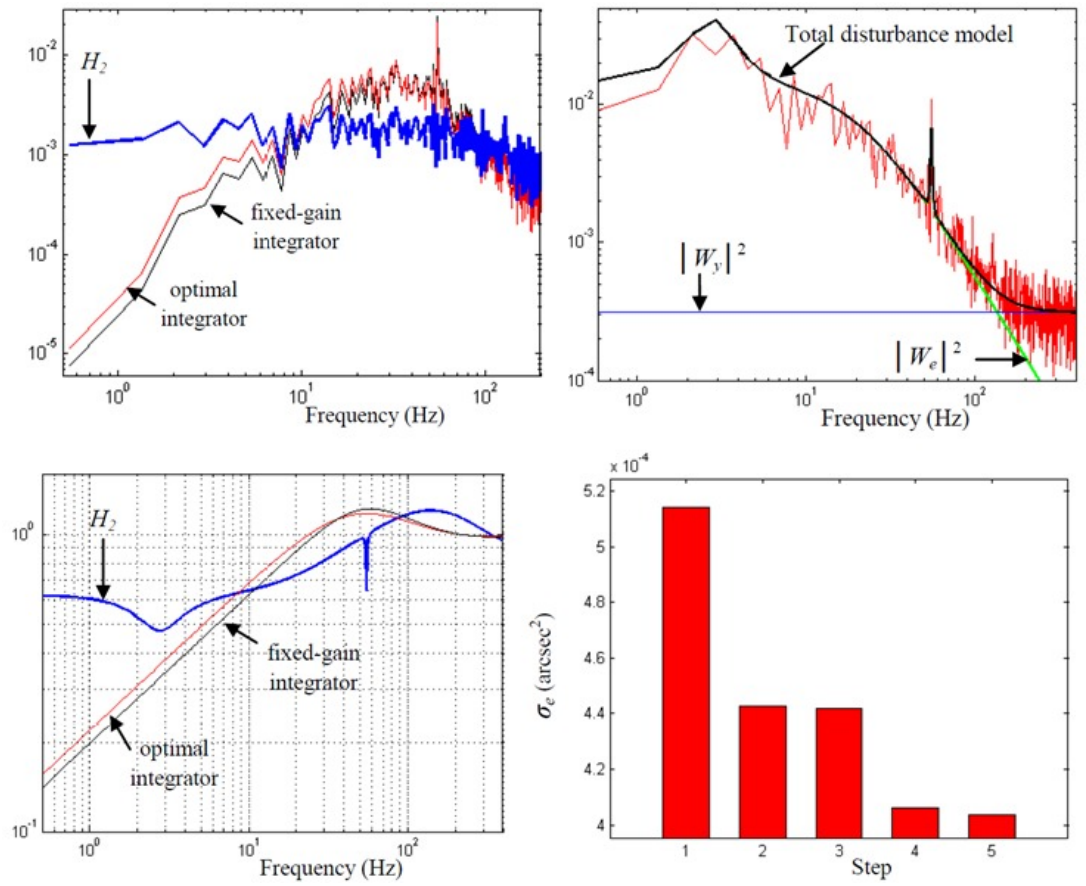

Figure 11. Left: scanning $F$ in search for further vibrations (top) and value of the residual phase (bottom). Right: results for a fifth order controller (1 leaky integrator and 2 notches); top-left: residual PSD for the optimal turbulence model and PSDs of integrators; top-right: PSD of disturbances and noise for the minimum variance; bottom-left: sensitivity functions for integral and final $\mathrm{H}_{2}$ controllers; bottom-right: progressive reduction of residual variance for the sequence of design steps.

of the controller on the basis of spectral information. Time-domain identification methods could also be used for the design of model-based controllers. ${ }^{22}$ It is worth noting that at present systematic performance comparison between methods is lacking in the panorama of all that has been proposed for vibration mitigation. Yet, precise and realistic performance evaluation can be conducted in "replay," that is using only on-sky WFS data.

Throughout the know-how of AO users for the different telescopes we have presented, it seems clear that $\mathrm{AO}$ control allowing for vibration mitigation is to be considered. In addition, other perturbation sources enter a global disturbance. In Extremely Large Telescope, no one can say precisely how will look like all the disturbance's components. With this concern in mind, keeping a good flexibility of the control structure in the design of real time computers should be a good option to compensate for various forms of perturbations. In some cases, when stable energetic vibration peaks are present, notch filters can be used upstream from an AO controller with a more flexible structure. However, one has to keep in mind that an unnecessary large attenuation might degrade the performance of the whole control loop, because of Bode's constraint. ${ }^{24}$ From this point of view, an AO controller that compensates for the global disturbances should always be investigated for performance comparison.

For on-sky operations, an obvious issue is the update of such AO controllers: size of the batch used for the identification/design step, update frequency, relevance of real-time adaptive controllers. Answers are not so simple: the size should be long enough to lead to a meaningful model, but not too long, since otherwise statistical characteristics of the perturbation may change significantly between the beginning and the end of the buffer; ideally, the update frequency should be as high as possible, but a real-time adaptation poses the tricky problem of loop stability, while it should not be discarded if very efficient... From what was reported by several AO users, for medium seing conditions, a rather high rate seems to be an update every $10 \mathrm{~s}, 30 \mathrm{~s}$ for a medium rate and $\sim 1 \mathrm{mn}$ might be a little bit slow. Updates, if too slow, may also lead to "bumps" in the actuator's trajectories, and thus to possible dents in performance. As for real-time considerations, these advanced controllers are likely to be used on a limited number of modes only, so that their complexity should not be a limiting factor. 


\section{REFERENCES}

1. G. Rousset, F. Lacombe, P. Puget, N. N. Hubin, E. Gendron, T. Fusco, R. Arsenault, J. Charton, P. Feautrier, P. Gigan, P. Y. Kern, A. Lagrange, P. Madec, D. Mouillet, D. Rabaud, P. Rabou, E. Stadler, and G. Zins, "NAOS, the first AO system of the VLT: on-sky performance," in Society of Photo-Optical Instrumentation Engineers (SPIE) Conference Series, P. L. Wizinowich \& D. Bonaccini, ed., 4839, pp. 140149, Feb. 2003.

2. C. Petit, J.-M. Conan, C. Kulcsár, H.-F. Raynaud, and T. Fusco, "First laboratory validation of vibration filtering with LQG control law for adaptive optics," Optics Express 16(1), pp. 87-97, 2008.

3. E. Fedrigo, R. Muradore, and D. Zilio, "High performance adaptive optics system with fine tip/tilt control," Control Engineering Practice 17(1), pp. 122-135, 2009.

4. S. Meimon, C. Petit, T. Fusco, and C. Kulcsár, "Tip-tilt disturbance model identification for Kalman-based control scheme: application to XAO and ELT systems," J. Opt. Soc. Am. A 27, pp. A122-A132, Nov 2010.

5. L. A. Poyneer and J.-P. Véran, "Kalman filtering to suppress spurious signals in adaptive optics control," J. Opt. Soc. Am. A 27(11), pp. A223-A234, 2010.

6. B. Neichel, F. Rigaut, A. Guesalaga, I. Rodriguez, and D. Guzman, "Kalman and H-infinity controllers for GeMS," in Optical Society of America on Adaptive Optics, jul 2011.

7. G. Agapito, F. Quirós-Pacheco, P. Tesi, A. Riccardi, and S. Esposito, "Observer-based control techniques for the LBT adaptive optics under telescope vibrations," European Journal of Control 17(3), pp. 316 - 326, 2011.

8. G. Sivo, C. Kulcsár, H.-F. Raynaud, J.-M. Conan, E. Gendron, and F. Vidal, "MOAO real-time LQG implementation on CANARY," in AO4ELT, (Victoria, Canada), 2011.

9. C. Correia, J.-P. Véran, and G. Herriot, "Advanced vibration suppression algorithms in adaptive optics systems," J. Opt. Soc. Am. A 29, pp. 185-194, Mar 2012.

10. A. Guesalaga, B. Neichel, F. Rigaut, J. Osborn, and D. Guzman, "Design of frequency-based controllers for vibration mitigation at the Gemini-South telescope," Society of Photo-Optical Instrumentation Engineers (SPIE) Conference Series 8447, jul 2012. This conference.

11. L. Petazzi, R. Muradore, E. Fedrigo, and M. R. Clare, "On the rejection of vibrations in adaptive optics systems," Society of Photo-Optical Instrumentation Engineers (SPIE) Conference Series 8447, 2012. This conference.

12. J.-P. Véran and L. Poyneer, "Characterization of the $\mathrm{T} / \mathrm{T}$ conditions at Gemini using adaptive optics telemetry data," in 1st AO4ELT Conference - Adaptative Optics for Extremely Large Telescopes proceedings, T. F. Y. Clénet, J.-M. Conan and G. Rousset, eds., AO4ELT, EDP Sciences, Jun 2009.

13. C. Correia, J.-P. Véran, and L. Poyneer, "Gemini Planet Imager minimum-variance tip-tilt controllers," in OSA Topical Meeting on Adaptive Optics: Methods, Analysis and Applications, p. AMB2, Optical Society of America, 2011.

14. E. Gendron, F. Vidal, M. Brangier, T. Morris, Z. Hubert, A. Basden, G. Rousset, R. Myers, F. Chemla, A. Longmore, T. Butterley, N. Dipper, C. Dunlop, D. Geng, D. Gratadour, D. Henry, P. Laporte, N. Looker, D. Perret, A. Sevin, G. Talbot, and E. Younger, "MOAO first on-sky demonstration with CANARY," Astronomy and Astrophysics 529(1), pp. 529L2.1-529L2.4, 2011.

15. J. M. Hill, "The Large Binocular Telescope," Appl. Opt. 49, pp. D115-D122, 2010.

16. S. Esposito, A. Riccardi, E. Pinna, A. T. Puglisi, and F. Quiros-Pacheco et al., "Natural guide stars adaptive optics system at LBT: FLAO commissioning and science operations status," Society of PhotoOptical Instrumentation Engineers (SPIE) Conference Series 8447, 2012. This conference.

17. S. Esposito, A. Riccardi, L. Fini, and E. Pinna et. al., "The LBT AO system on-sky results," in 2nd AO4ELT Conference - Adaptive Optics for Extremely Large Telescopes proceedings, 2011.

18. H. Bonnet, B. Bauvir, A. Wallander, M. Cantzler, J. Carstens, F. Caruso, N. di Lieto, S. Guisard, P. Haguenauer, N. Housen, M. Mornhinweg, J.-L. Nicoud, A. Ramirez, J. Sahlmann, G. Vasisht, S. Wehner, and J. Zagal, "Enabling Fringe Tracking at the VLTI," The Messenger 126, pp. 37-40, Dec. 2006.

19. F. Martinache, O. Guyon, S. C. Clergeon, and V. Garrel, "The Subaru coronagraphic extreme AO project: first observations," Society of Photo-Optical Instrumentation Engineers (SPIE) Conference Series 8447, 2012. This conference. 
20. O. Guyon, T. Matsuo, and R. Angel, "Coronagraphic low order wavefront sensor: Principle and application to a phase-induced amplitude coronagraph," ApJ 693, pp. 75-84, 2009.

21. F. P. A. Vogt, F. Martinache, O. Guyon, T. Yoshikawa, K. Yokochi, V. Garrel, and T. Matsuo, "Coronagraphic low-order wavefront sensor: Postprocessing sensitivity enhancer for high-performance coronagraphs," PASP 123, pp. 1434-1441, 2011.

22. C. Kulcsár, P. Massioni, G. Sivo, and H.-F. Raynaud, "Vibration mitigation in adaptive optics control," Society of Photo-Optical Instrumentation Engineers (SPIE) Conference Series 8447, 2012. This conference.

23. J. Doyle, K. Glover, P. Khargonekar, and B. Francis, "State-space solutions to standard $H_{2}$ and $H_{\infty}$ control problems," Automatic Control, IEEE Transactions on 34, pp. 831 -847, aug 1989.

24. C. Mohtadi, "Bode's integral theorem for discrete-time systems," in Proc. IEE, 137, pp. 57-66, 1990. 